\title{
Adaptation of the 2015 American College of Rheumatology treatment guideline for rheumatoid arthritis for the Eastern Mediterranean Region: an exemplar of the GRADE Adolopment
}

\author{
Andrea Darzi ${ }^{1}$, Manale Harfouche ${ }^{1}$, Thurayya Arayssi ${ }^{2}$, Samar Alemadii ${ }^{2}$ Khalid A. Alnaqbi ${ }^{3}$, Humeira Badsha ${ }^{4}$, \\ Farida Al Balushi ${ }^{5}$, Bassel Elzorkany ${ }^{6}$, Hussein Halabi ${ }^{7}$, Mohammed Hamoudeh ${ }^{8}$, Wissam Hazer ${ }^{9}$, Basel Masri ${ }^{10}$, \\ Mohammed A. Omair ${ }^{11}$, Imad Uthman ${ }^{12}$, Nelly Ziade ${ }^{13}$, Jasvinder A. Singh ${ }^{14,15}$, Robin Christiansen ${ }^{16}$, \\ Peter Tugwell ${ }^{17}$, Holger J. Schünemann ${ }^{18,19}$ and Elie A. Akl ${ }^{1,19,20^{*}}$
}

\begin{abstract}
Background: It has been hypothesized that adaptation of health practice guidelines to the local setting is expected to improve their uptake and implementation while cutting on required resources. We recently adapted the published American College of Rheumatology (ACR) Rheumatoid Arthritis (RA) treatment guideline to the Eastern Mediterranean Region (EMR). The objective of this paper is to describe the process used for the adaptation of the 2015 ACR guideline on the treatment of RA for the EMR.

Methods: We used the GRADE-Adolopment methodology for the guideline adaptation process. We describe in detail how adolopment enhanced the efficiency of the following steps of the guideline adaptation process: (1) groups and roles, (2) selecting guideline topics, (3) identifying and training guideline panelists, (4) prioritizing questions and outcomes, (5) identifying, updating or conducting systematic reviews, (6) preparing GRADE evidence tables and EtD frameworks, (7) formulating and grading strength of recommendations, (8) using the GRADEpro-GDT software.

Results: The adolopment process took 6 months from January to June 2016 with a project coordinator dedicating 40\% of her time, and the two co-chairs dedicating $5 \%$ and $10 \%$ of their times respectively. In addition, a research assistant worked $60 \%$ of her time over the last 3 months of the project. We held our face-to-face panel meeting in Qatar. Our literature update included five newly published trials. The certainty of the evidence of three of the eight recommendations changed: one from moderate to very low and two from low to very low. The factors that justified a very low certainty of the evidence in the three recommendations were: serious risk of bias and very serious imprecision. The strength of five of the recommendations changed from strong to conditional. The factors that justified the conditional strength of these 5 recommendations were: cost $(n=5[100 \%])$, impact on health equities $(n=4$ [80\%]), the balance of benefits and harms $(n=1$ [20\%]) and acceptability $(n=1$ [20\%]).

(Continued on next page)
\end{abstract}

\footnotetext{
* Correspondence: ea32@aub.edu.lb

${ }^{1}$ AUB GRADE Center, Clinical Research Institute, American University of Beirut,

PO Box 11-0236, Riad El Solh, Beirut 1107 2020, Lebanon

${ }^{19}$ Department of Health Research Methods, Evidence, and Impact (HE\&l),

McMaster University, Hamilton, ON, Canada

Full list of author information is available at the end of the article
} 
(Continued from previous page)

Conclusion: This project confirmed the feasibility of GRADE-Adolopment. It also highlighted the value of collaboration with the organization that had originally developed the treatment guideline. We discuss the implications for both guideline adaptation and future research to advance the field.

Keywords: Practice guideline, Adaptation, GRADE, Evidence-based medicine, Eastern Mediterranean Region, Rheumatoid arthritis, Conflicts of interest

\section{Background}

Guidelines are considered an integral aspect of the development of a standardized high quality health care using evidence-based practices [1]. Guidelines are defined by the World Health Organization (WHO) as: "systematically developed evidence-based statements which assist providers, recipients and other stakeholders to make informed decisions about appropriate health interventions" [2].

Development of guidelines de novo faces multiple challenges including financial and human resource requirements and time constraints [3]. On the other hand, using guidelines developed for one setting in another one (i.e., guideline adoption) may be inappropriate due to differing contextual factors such as acceptability or feasibility of the proposed intervention.

Adaptation of guidelines would address the above challenges and limitations, through modifying the recommendations to account for contextual factors. It has been hypothesized that adaptation of guidelines to the local setting is expected to improve their uptake and implementation [4]. One of the challenges of the process of adapting guidelines is to keep it efficient while ensuring it is evidence-based.

A recently published survey identified eight methods for guidelines adaptation, one of which is GRADE- Adolopment [5]. Adolopment combines the advantages of adoption, adaptation and de novo guideline development and is based on three cornerstones: (1) identifying and prioritizing credible existing guidelines or evidence syntheses that are of both interest and relevance; (2) evaluating and completing the GRADE Evidence to Decision (EtD) frameworks for each of the recommendations [6]; and (3) deciding on a final adoption, adaptation or de novo development for each of the recommendations [7].

We adoloped the recently published American College of Rheumatology (ACR) Rheumatoid Arthritis (RA) treatment guideline to the Eastern Mediterranean Region [8]. This project was a collaborative effort between the Weill Cornell Medical College in Qatar, the Middle East Rheumatoid Arthritis Consortium (MERAC), and the American University of Beirut (AUB) GRADE Center.

The objective of this paper is to describe the process used for the adolopment of the 2015 ACR guideline on the treatment of RA for the Eastern Mediterranean
Region. We report on the specific recommendations resulting from this process in a separate paper.

\section{Methods}

We used the adolopment methodology to adapt the 2015 ACR RA treatment guideline [8] to the Eastern Mediterranean Region and based the process on the GRADEAdolopment approach [7]. Shortly after the publication of the ACR RA guidelines, (which we refer to as the source guideline'), the MERAC group identified them as a priority for adaptation. The source guideline [8] used the GRADE methodology to rate the certainty of evidence. We obtained the approval of the ACR senior director of quality for using that guideline in our project.

We structured the process for the 'adoloped guideline' using the Guidelines 2.0 comprehensive checklist for guideline development, which consists of 18 steps [9]. Table 1 highlights what steps were transferred from the process of the source guideline, and what steps we specifically conducted for the current process. Table 2 on the other hand presents how the guideline process matches the 18 steps outlined in the 'Guidelines 2.0' comprehensive checklist for guideline development. We provide below a detailed description of how adolopment enhanced the efficiency of the following steps compared to de novo guideline development:

1. Groups and roles

2. Selecting guideline topics

3. Identifying and training guideline panelists

4. Prioritizing questions and outcomes

5. Identifying, updating or conducting systematic reviews

6. Preparing GRADE evidence tables and EtD frameworks

7. Formulating and grading strength of recommendations

8. Using the GRADEPro-GDT software

\section{Groups and roles}

This step included identifying and recruiting individuals for the following three groups: a guideline executive committee, a guideline coordination team, and a guideline panel (see Table 1). A content expert (rheumatologist) and a guideline methodologist co-chaired the final panel 


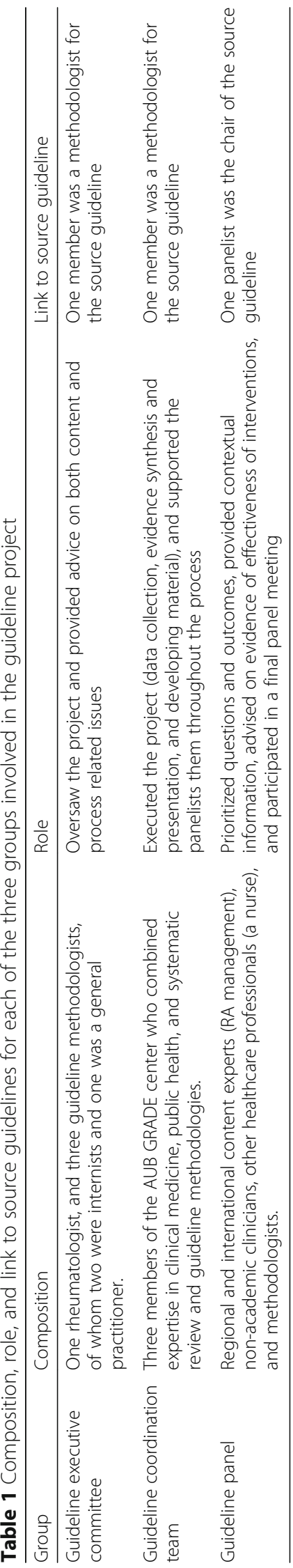




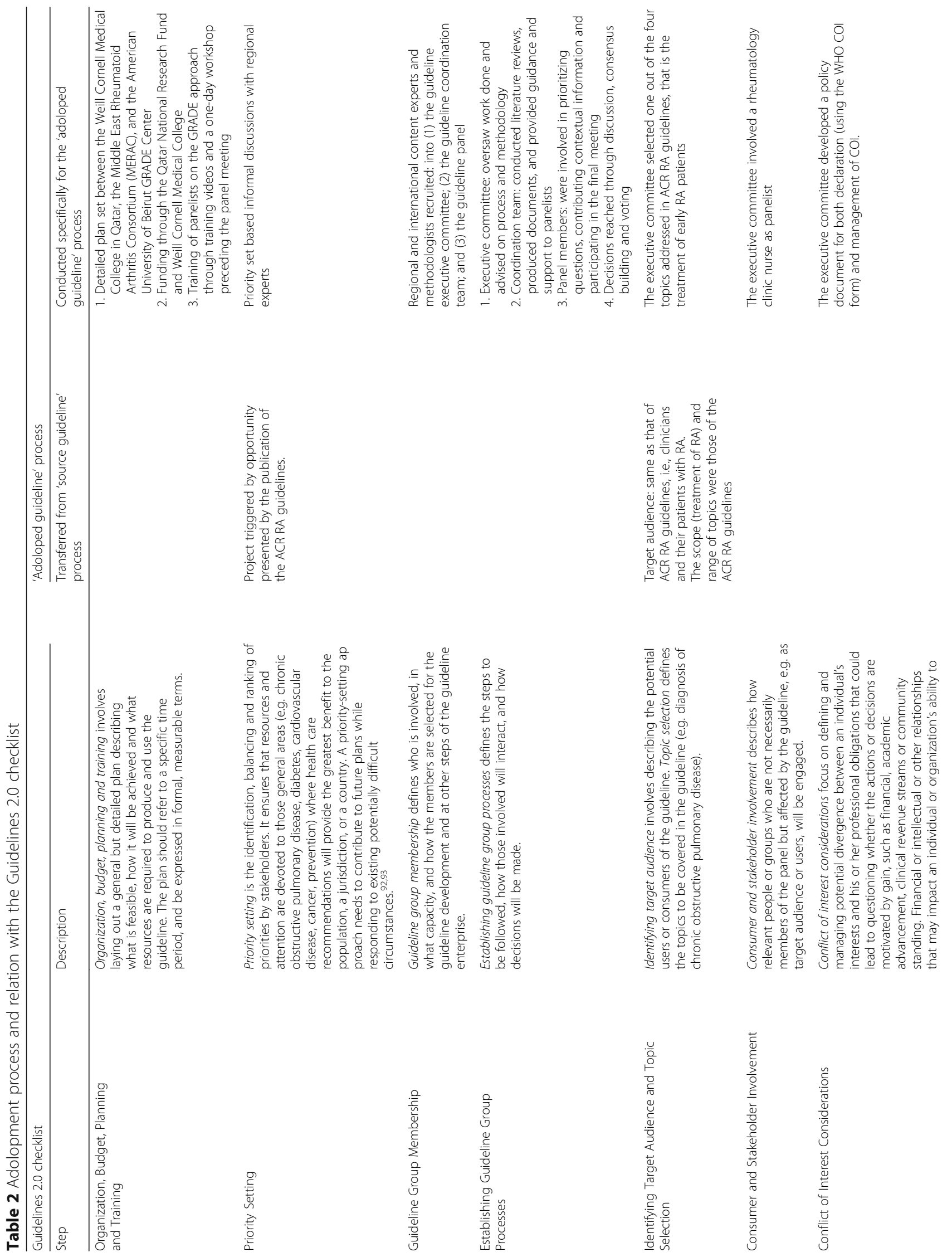



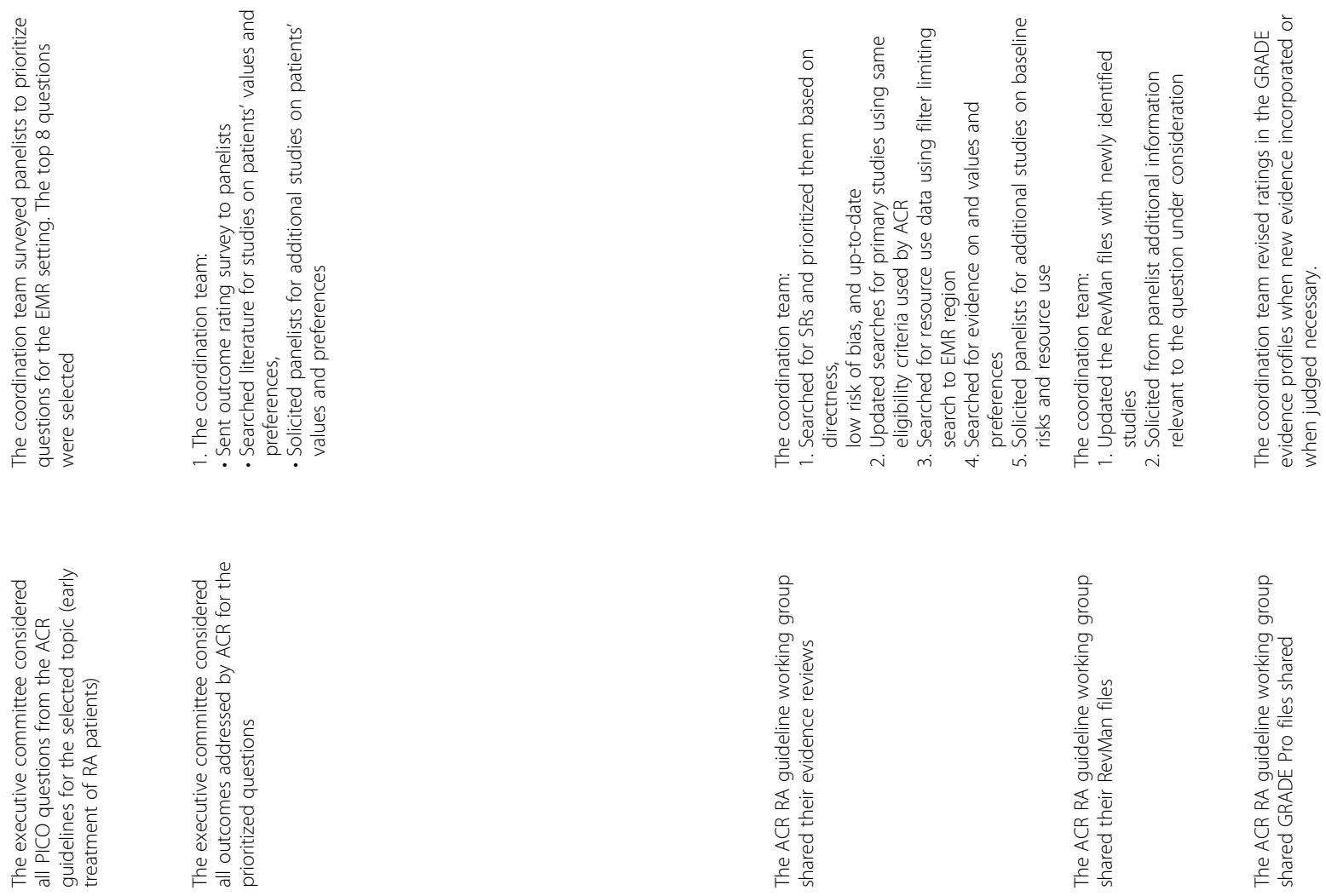

을.

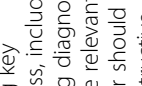

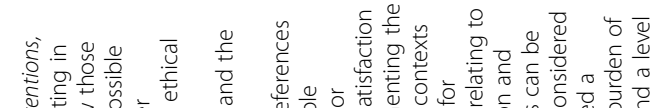

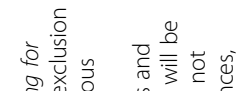

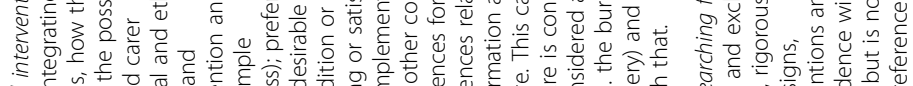

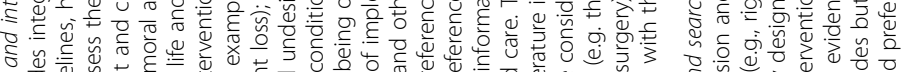

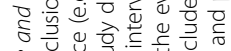

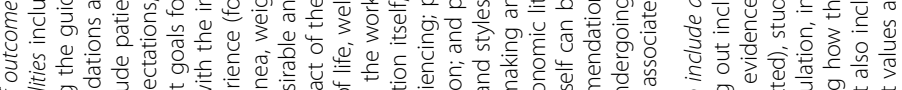

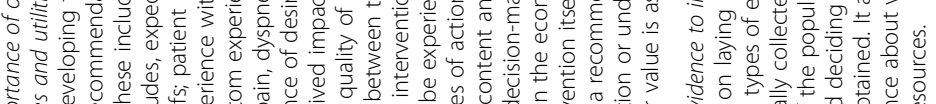

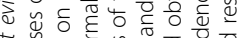

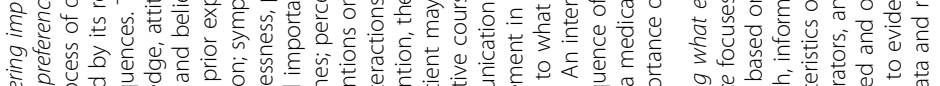

$\begin{array}{lll} & \\ 0 & & \\ 0\end{array}$

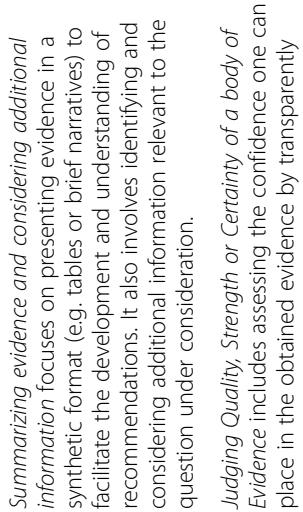

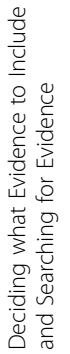

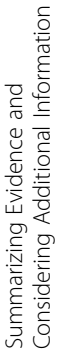

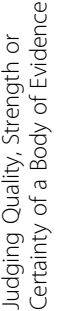




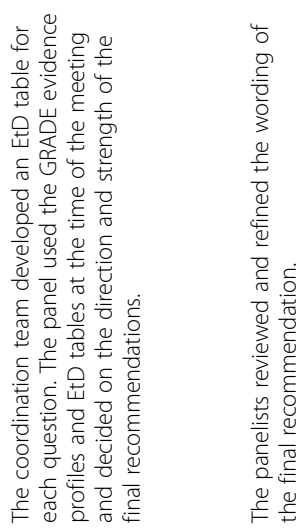

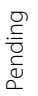

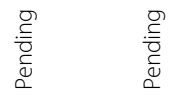

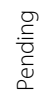

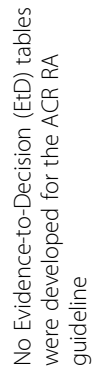

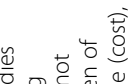

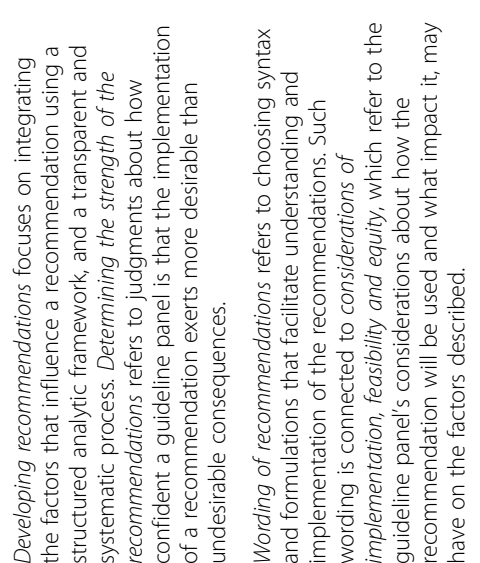

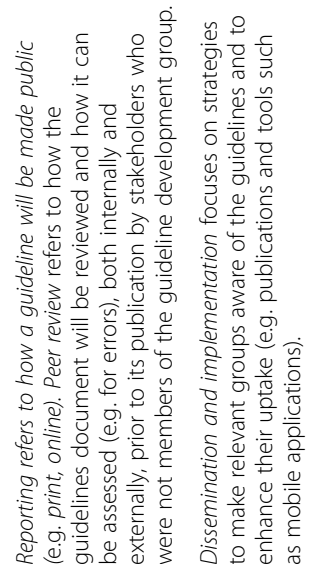

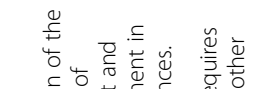

出.

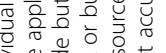

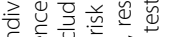

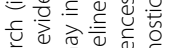

政

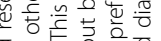

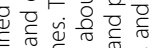

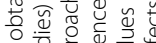

\&

ऊ) 0

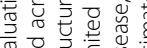

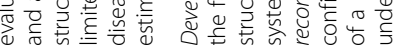

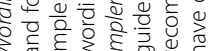

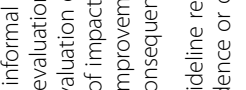

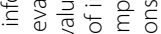

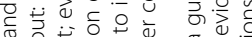

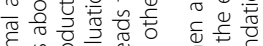

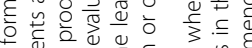

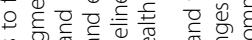

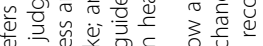

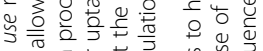

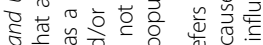

๖.ّ人
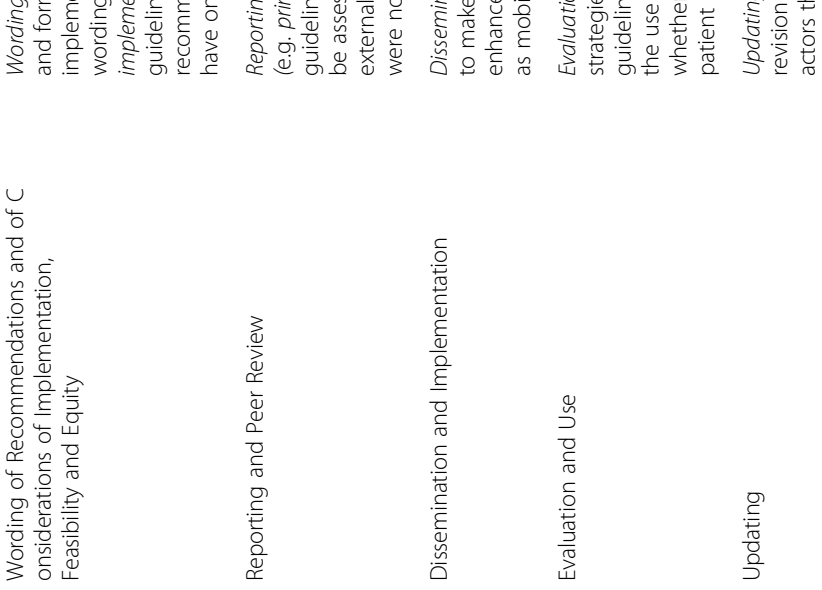

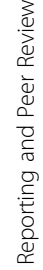

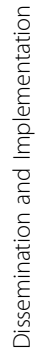

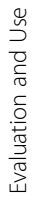


meeting. They facilitated and steered the discussion, reflected on and summarized the panelists viewpoints, raised issues/concerns that could inform the decisionmaking process; and attempted to achieve consensus whenever possible. The methodologist co-chair did not vote while the content co-chair did.

In term of conflicts of interest (COI) rules, we asked the panel members to fill out an online COI declaration form, adopted from the World Health Organization. We will include these forms as an appendix in the adopted guideline manuscript. All panelists received a COI policy document explaining the approach to managing conflicts during the guideline process. The two panel co-chairs exercised the rule of "no strong advocacy" to minimize the effect of the COIs. Accordingly, panelists were invited to share their opinions and positions on each recommendation, while avoiding repetition or being verbally forceful. The co-chairs had the right to exclude panel members with COI from discussions or decisionmaking on recommendations they were conflicted on.

\section{Selecting guideline topics}

The source guideline (the 2015 ACR RA treatment guideline) addressed four different topics: (i) management of patients with early RA (15 questions), (ii) management of patients with established RA (44 questions), (iii) management of patients with established RA with high risk (24 questions), and (iv) live vaccines in early or established RA patients (5 questions). Given the limited time and resources, the guideline executive committee chose to address a set of prioritized questions from the first topic, i.e., management of patients with early RA. While this choice was pragmatic it also aimed to produce a coherent set of questions, i.e., within the same type of patient population.

\section{Identifying and training guideline panelists}

The executive committee recruited guideline panelists in a way to represent a diverse set of expertise. Panelists included regional and international rheumatologists, a nurse practicing in Qatar, and methodologists from AUB GRADE and the McMaster GRADE (Mac GRADE) centers. The regional rheumatologists were members of the Middle East Rheumatoid Arthritis Consortium (MERAC). MERAC is based at the Weill Cornell Medical College in Qatar and is comprised of rheumatologists from Jordan, Kingdom of Saudi Arabia, Lebanon, United Arab Emirates, and Qatar. MERAC's research focuses on studying rheumatoid arthritis in the Middle East.

One month ahead of the panel meeting, we shared with the panelists training videos on the GRADE approach for guideline development found on the webpage of the MacGRADE center. Also, the first day of the guideline meeting consisted of a workshop on the use of the GRADE Adolopment methodology. The workshop used material related to the one of the planned guideline questions.

\section{Prioritizing questions and outcomes}

The core team at the AUB GRADE Center developed a prioritization survey that included all questions from the source guideline addressing the prioritized topic, i.e., early RA. We asked the panelists to rate the importance of these questions on a Likert scale of 1-5 (least - most important), from the perspective of patients in the EMR. We selected 8 questions taking into consideration panelists' ratings and the coherence amongst those questions. We communicated the list of selected questions to the panelists for approval. It was not possible afterwards to change or add questions to the project.

Then, we sent the panelists a survey to prioritize patient important outcomes relating to the selected questions. The survey specifically asked participants to "consider outcomes that might be important to someone making a decision to use or not to use the treatment". The panelists used a Likert scale of 1-9 to rate the importance of these outcomes for making a decision as follows: (1-3) not important; (4-6) important but not critical; and (7-9) critical.

\section{Identifying, updating or conducting systematic reviews}

Figure 1 depicts the process of searching and using the identified evidence for the recommendation questions selected by the panel. We ran two searches for systematic reviews and primary studies respectively. We searched Medline, Embase, Cochrane and Epistemonikos electronic databases from the last search date of the source guideline in September 2014, till February 2016. We used the same search terms as the source guideline search; we only added study design filters for primary studies and systematic reviews respectively. The search terms included both medical subject headings (MeSH) and text words.

We used standards systematic review methodology including duplicate and independent approach to title and abstract screening, full text screening, and data abstraction. We conducted calibration exercises, used standardized and pilot tested forms, and relied on a third reviewer to resolve disagreements.

When evaluating the potential use of identified systematic review, we considered the following three characteristics as important:

- Relevance (directness): we assessed the relevance of identified systematic reviews by matching their PICO to the PICO of the guideline questions. The minimum requirement was for the Population, 


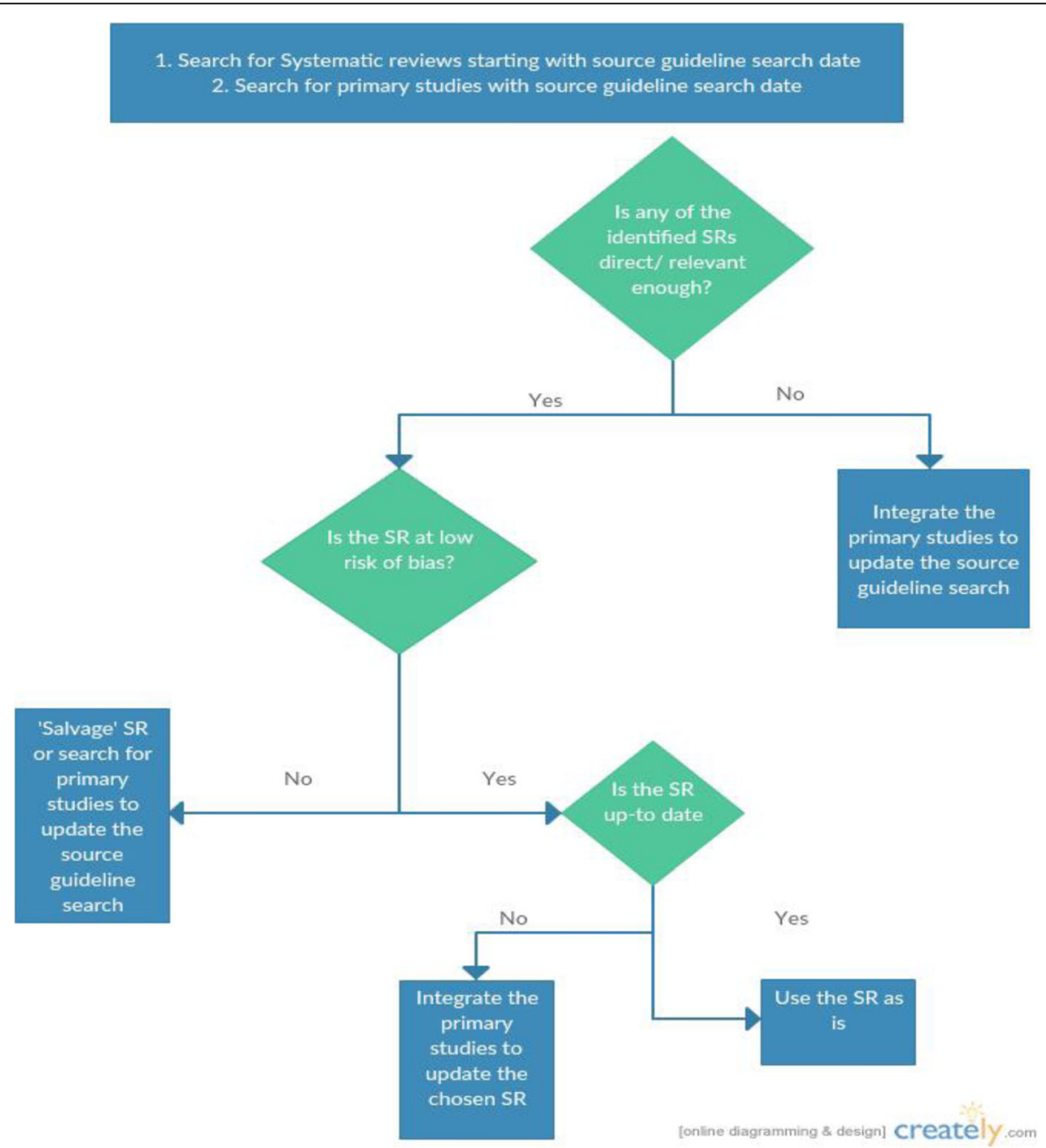

Fig. 1 Algorithm of our search and use of the identified evidence. *Salvaging the Systematic Review would entail redoing the deficient part of the methods (e.g. meta-analysis section)

Intervention and Control elements to match to a reasonable degree, i.e., not to have serious indirectness for more than one of the three elements.

- Quality (risk of bias): we assessed the risk of bias of relevant systematic reviews using AMSTAR [10]. If we identified more than one relevant systematic review we prioritized the one with the highest quality.

- Being Up to date: we assessed whether the systematic review judged to be relevant and of highest quality was up to date. In case we had identified more than one systematic review, the judgment of relative up-to-dateness would have considered whether the systematic reviews included all relevant studies. When we identified new primary studies, we integrated the findings in the chosen systematic review.
When we identified no usable systematic review (based on the three above criteria), we updated the systematic review conducted by the source guideline-working group using the results of the search for primary studies.

In addition, we searched the literature for studies and data relevant to patients' values and preferences and economic data. The eligibility criteria related to both the population of interest (rheumatoid arthritis) and the interventions of interest. We searched Medline using both medical subject headings $(\mathrm{MeSH})$ and text words related to 'rheumatoid arthritis' and the interventions of interest. We used a filter-limiting search to EMR region when searching for economic data but not when searching for values and preferences. Also, we limited to a period covering the last 10 years when searching for values and preferences. In addition, we solicited panelists for additional studies on baseline risks and economic data. 


\section{Preparing GRADE evidence tables and EtD frameworks}

In preparation for the guideline panel meeting, we used GRADEpro-GDT guideline development tool (www.gradepro.org) to develop for each recommendation question the standard tables proposed by the GRADE working group to facilitate the process [11]:

1. Evidence Tables: they provide for each of the outcomes of interest a summary of the synthesized evidence (ideally based on a meta-analysis) and the rating of the certainty of the evidence. The GRADE working group has developed two versions of these evidence tables: Evidence Profiles (EP) (a more detailed version) and Summary of Findings tables (SoF) (a less detailed version) [12];

2. Evidence to Decision frameworks (EtD): they are intended to facilitate the panel's decision-making process for going from evidence to recommendation by summarizing in a structured and transparent way the evidence for the following factors: benefits and harms, values and preferences, cost, cost effectiveness, equity, acceptability and feasibility [6, 13]. As the source guideline did not include EtD frameworks, we developed our own for this project.

The team that conducted the systematic review for the source guideline, shared with us, with the approval of ACR senior director of quality, files relevant to the recommendation questions of interest (e.g., Review Manager and GRADEpro-GDT files).

\section{Formulating and grading strength of recommendations}

The guideline panelists revised the Evidence Profiles and provided input prior to the panel (face-to-face) meeting. During the panel meeting, we used the GRADE Evidence-to-Decision frameworks to assist the panel in formulating and grading the final recommendations. During this process, the panel decided to downgrade the evidence for three recommendations from low to very low due to a judgment for the imprecision factor that was different from that of the source guideline.

We intentionally did not expose the panelists to the recommendations from the source guideline prior to finalization of the recommendations. For each of these recommendations, we expected the use of the adolopment process to lead in one of three potential outcomes:

- Adoption of the recommendation, i.e., the use of the original recommendation as is;

- Adaptation of the recommendation, i.e., the modification of the original recommendation;

- De novo development of the recommendation, i.e., the creation of a new recommendation.
Using the GRADEpro-GDT software (www.Gradepro.Org) We used the GRADEpro-GDT software to conduct the following tasks:

- Collect the COI disclosures statements of panelists

- Update the evidence profiles and develop EtD frameworks for each of the guideline's questions;

- Facilitate the panel discussions by projecting the tables in real time during the meeting;

- Export tables in word format to include in the final guideline report.

\section{Results}

The adolopment process yielded eight recommendations, which will be detailed in another paper. Below we provide a description relating to the: (1) timeframe of the process, (2) results of the literature review, and (3) change in the certainty of evidence and the strength of recommendation.

\section{Timeframe}

Table 3 provides a detailed description of the timeframe for the different steps of the adolopment process. The process took 6 months starting January 2016 with a project coordinator (AD) dedicating 40\% of her time, and the two co-chairs (TA, EAA) dedicating $5 \%$ and $10 \%$ of their times respectively. In addition, a research assistant $(\mathrm{MH})$ worked $60 \%$ of her time over the last 3 months of the project. We dedicated the first 2 months to setting up the project, including drafting the project overview, selecting and inviting the panelists, and collecting their declarations of conflicts of interest (COI). The most time consuming steps were: (1) deciding what evidence to include and searching for evidence (2) summarizing the evidence and considering additional information and (3) judging strength or certainty of a body of evidence. We completed these steps over a period of 3 months. To make the process more efficient we ran these steps in parallel. Once these steps were finalized we presented the synthesized evidence in a three-day face-to-face panel meeting in Qatar in May 2015.

\section{Results of the literature search}

Table 4 describes the results of the systematic literature search per question. The search for systematic reviews of effectiveness, with no date limit, yielded 772 papers, two of which were relevant to the project $[14,15]$. The search for primary studies of effectiveness done after the search date for the ACR RA treatment guideline yielded 2051 papers, five of which were eligible [16-20].

With regards to our search for studies on patients' values and preferences, we identified 16 relevant studies 
Table 3 Timeframe for the different steps of the process (January-June 2016)

\begin{tabular}{|c|c|c|c|c|c|c|c|c|c|c|}
\hline & January & January & February & February & March & March & April & April & May & May \\
\hline Organization, Budget, Planning and Training & $\times$ & $\times$ & & & & & & & & \\
\hline Priority Setting & $\times$ & $\times$ & & & & & & & & \\
\hline Guideline Group Membership & & & $\times$ & & & & & & & \\
\hline Establishing Guideline Group Processes & & & $\times$ & & & & & & & \\
\hline Identifying Target Audience and Topic Selection & & & & $x$ & & & & & & \\
\hline Consumer and Stakeholder Involvement & & & & $x$ & & & & & & \\
\hline Conflict of Interest Considerations & & & & $\times$ & & & & & & \\
\hline (PICO) Question Generation & & & & & $x$ & & & & & \\
\hline $\begin{array}{l}\text { Considering Importance of Outcomes and Interventions, Values, } \\
\text { Preferences and Utilities }\end{array}$ & & & & & & $\times$ & $x$ & & & \\
\hline Deciding what Evidence to Include and Searching for Evidence & & & & & & $\times$ & $x$ & & & \\
\hline Summarizing Evidence and Considering Additional Information & & & & & & & & $\times$ & $\times$ & \\
\hline Judging Quality, Strength or Certainty of a Body of Evidence & & & & & & & & $\times$ & $x$ & \\
\hline Developing Recommendations and Determining their Strength & & & & & & & & & & $\times$ \\
\hline $\begin{array}{l}\text { Wording of Recommendations and of Considerations of } \\
\text { Implementation, Feasibility and Equity }\end{array}$ & & & & & & & & & & $x$ \\
\hline
\end{tabular}

but none were specific to the Eastern Mediterranean Region context. The information we retrieved looked at outcome valuation and medication preference in general (i.e., not specific to our questions). We did not identify any studies on resource use relevant to the Eastern Mediterranean Region.

\section{Change in the certainty of evidence and the strength of recommendation}

After we formulated the eight final recommendations, we compared the certainty and strength of each of the adoloped recommendations to corresponding recommendations from the source guideline. The certainty of the evidence of three of the eight recommendations changed: one from moderate to very low and two from low to very low. The factors that

Table 4 The number of included studies per question resulting from different literature searches

\begin{tabular}{lllll}
\hline $\begin{array}{l}\text { Question } \\
\text { number }\end{array}$ & $\begin{array}{l}\text { Studies included in } \\
\text { source guideline }\end{array}$ & $\begin{array}{l}\text { SR } \\
\text { search }\end{array}$ & $\begin{array}{l}\text { Screening of SR } \\
\text { for primary } \\
\text { studies }^{\mathrm{a}}\end{array}$ & $\begin{array}{l}\text { Primary } \\
\text { study } \\
\text { search }\end{array}$ \\
\hline Question 1 & - & 1 & 2 & 1 \\
Question 2 & 3 & 0 & - & 0 \\
Question 3 & 4 & 0 & - & 0 \\
Question 4 & 7 & 1 & 2 & 3 \\
Question 5 & 0 & 0 & - & 2 \\
Question 6 & 1 & 0 & - & 1 \\
Question 7 & 1 & 0 & - & 0 \\
Question 8 & 1 & 0 & - & 0 \\
\hline
\end{tabular}

$S R$ systematic review

aprimary studies included in the identified systematic review and not included in the source guideline search justified a very low certainty of the evidence in these three recommendations were: serious risk of bias and very serious imprecision. The strength of five out of the eight recommendations changed from strong to conditional. The factors that justified the conditional strength of these 5 recommendations were the following: cost $(n=5)$, impact on health equities $(n=4)$, the balance of benefits and harms $(n=1)$ and acceptability $(\mathrm{n}=1)$.

\section{Discussion}

We describe in this paper the process used for the adaptation of the 2015 ACR RA treatment guideline for the EMR. The process, which took 6 months was based on the GRADE-Adolopment approach and resulted in a total of 8 recommendations on the management of the early RA. The strength of five out of the eight recommendations changed from strong to conditional.

This project confirmed the feasibility of GRADEAdolopment [7]: (1) Use of existing evidence syntheses for every recommendation question (2); short time frame for completing the guideline (about 6 months) thanks to using existing systematic reviews and collaborating with the source guideline organization as detailed below; and (3) use of a transparent and structured process in formulating the recommendation.

Our collaboration with the source guideline organization proved to be crucial for the success of the project. A major contributing factor was the willingness of the senior director of quality for the ACR to allow the unrestricted use of their recently published guideline as the basis for the adolopment 
process. As a result, we used ACR RA treatment guideline recommendation questions as a starting point to prioritize our questions; the participation of the chair and the methodologist of the ACR treatment guideline gave the panel unique insight into the source guideline's panel decision-making process; it helped with clarifying any uncertainties regarding the evidence synthesis and formulation of the final recommendations. In addition, the fact that the two guideline efforts used the same methodology (i.e., GRADE), and the same the tools (e.g., RevMan, GRADEPro-GDT) made our process more efficient.

This project was not without any challenges or barriers. One major challenge was the scarcity of evidence for contextual factors such as values and preferences, and economic data. The panel relied on its members' expert opinion to judge those factors as part of the EtD. Another challenge was the need to develop a plan (as describe above) to build the capacity of the panelists and of some members of the technical team in guideline development and adolopment. We were also challenged with the relatively short timeframe of 6 months. We managed by employing efficient processes, having the members of the core team dedicate a larger amount of their time to the project while limiting the number of questions to address.

To keep adolopment of guidelines efficient, guideline developers might need to accept a number of restrictions based on our experience:

- Accepting definitions or classifications used by the source guidelines for the condition, the interventions, and the outcomes

- Using either the exact same question or a question with narrower scope, compared to the source guidelines. Otherwise, the adapting group must run a new search strategy to ensure capturing all studies relevant to their new question

- Accepting the outcomes and the associated timeframes as prioritized by the source guidelines

- Accepting any limitations in the process of the source guidelines related to the systematic review process (e.g., search strategy, risk of bias assessment, rating of the certainty of evidence).

Having considered the above, it is also ideal that the guideline adolopment panel makes its own judgments about context sensitive aspects of the decision-making process:

- The indirectness of the evidence as it relates to the local population (i.e., the local population might be different from the population in the original setting and from participants included in trials);
- The indirectness of the evidence as it relates to the intervention (e.g., it might not be logistically possible to locally reproduce the 'treat to target' strategy in a way that is similar to what the relevant trials did and to what is available in the source setting);

- The baseline risk of the outcomes of interest (e.g., incidence of malignancies higher in the local population compared to the source setting)

- The values that the local population attach to the outcomes of interest (e.g., reduction in side effects) and their preferences for the interventions of interest (e.g., intravenously administered biologics);

- Contextual factors of the EtD, including priority of the problem, cost, cost effectiveness, impact on equity, acceptability and feasibility.

In terms of implications for future research, the adaptation process allowed us to highlight the scarcity of regional data related to rheumatoid arthritis when looking at contextual factors such as cost, cost effectiveness and values and preferences. This should encourage researchers to address these gaps through conducting primary studies. This will allow the adaptation process to be better contextualized and the data on which we rely on to be more systematic and evidence based rather than solely relying on the panels knowledge and opinions.

Schünemann et al. described a vision of guideline development through an "international collaboration with common aims and free of proprietary influences" [21]. Several of the concepts highlighted in the statement are highly relevant to a successful adaptation efforts. (1) Globalizing the evidence through a standardized database of existing evidence and gaps to serve as a shared resource for participating organizations; organizations interested in adapting guidelines could tap into this database to identify relevant source evidence summaries and evidence to decision frameworks. (2) Undertaking collaborative evidence reviews relevant to recommendation questions; while the source guideline working group develop the base evidence summaries based on high certainty systematic reviews, the adaptation working groups could contribute to their subsequent update. (3) Maintaining a collaborative network of organizations with common interests. This would allow a more efficient and effective coordination of efforts and allocation of resources [21].

\section{Conclusion}

It is important for major guideline developers interested in others building on their guidelines, to develop them in a manner that facilitates the later adaptation process. This includes making explicit the methods used in the evidence synthesis, evidence grading and 
recommendation development processes to allow reproducibility. It also includes making the products of those processes available to other groups (search strategies, data files, grade tables). Finally, making explicit the judgments and underlying justifications on the EtD factors would make it easier to other groups to make their own judgments.

\section{Abbreviations}

ACR: American College of Rheumatology; AUB: American University of Beirut; COI: Conflict of Interest; EMR: Eastern Mediterranean Region; EtD: Evidence to Decision; GDT: guideline development tool; MERAC: The Middle East Rheumatoid Arthritis Consortium; RA: Rheumatoid Arthritis; SoF: Summary of findings; WHO: World Health Organization

\section{Acknowledgements \\ The authors would like to acknowledge Ms. Hadeel Ashour, Ms. Soha Dargham, Ms. Marianthi Kapiri, Ms. Diana Jubeily and Dr. Karim B. Mohamed for their support in the organization of the meeting, taking minutes and facilitating communications amongst the participants. Also we would like to thank Ms. Amy Miller, Dr. Raveendhara Bannuru and Dr. Timothy McAlindon from the American College of Rheumatology Rheumatoid Arthritis guideline team. Finally, we would like to acknowledge the Parker Institute, Bispebjerg and Frederiksberg Hospital (R. Christensen) that was supported by a core grant from the Oak Foundation (OCAY-13-309).}

\section{Funding}

The Qatar National Research Fund played no role in design of the study and collection, analysis, and interpretation of data or in writing the manuscript. Weill Cornell Medical College in Qatar peer reviewed the article in the final stages.

\section{Availability of data and materials}

Not applicable

\section{Authors' contributions}

Conception and design: EAA, AJD, HJS. Data acquisition: EAA, AJD, MH. Data analysis: EAA, AJD. Interpretation of results: EAA, AJD, HJS. Manuscript drafting: EAA, AJD. Critically revision of the manuscript and approval of the final version: EAA, AJD, HJS, MH, TA, SA, KAA, HB, FB, BE, HH, MH, WH, BM, $M O, I U, N Z, J A S, R C$, PT. All authors read and approved the final manuscript

\section{Ethics approval and consent to participate}

Not applicable

\section{Consent for publication}

Not applicable

\section{Competing interests}

The authors report intellectual conflict of interest being members of the GRADE working group (AJD, RC, JAS, HJS, EAA) and authors of papers reporting adaptation approaches (AJD, HJS, EAA). JAS has received research grants from Takeda and Savient and consultant fees from Savient, Takeda, Regeneron, Merz, Iroko, Bioiberica, Crealta and Allergan pharmaceuticals, WebMD, UBM LLC and the American College of Rheumatology. JAS serves as the principal investigator for an investigator-initiated study funded by Horizon pharmaceuticals through a grant to DINORA, Inc., a 501 (c)(3) entity. JAS is a member of the executive of OMERACT, an organization that develops outcome measures in rheumatology and receives arms-length funding from 36 companies; a member of the American College of Rheumatology's (ACR) Annual Meeting Planning Committee (AMPC); Chair of the ACR Meet-theProfessor, Workshop and Study Group Subcommittee; and a member of the Veterans Affairs Rheumatology Field Advisory Committee.

\section{Publisher's Note}

Springer Nature remains neutral with regard to jurisdictional claims in published maps and institutional affiliations.

\section{Author details}

AUB GRADE Center, Clinical Research Institute, American University of Beirut, PO Box 11-0236, Riad El Solh, Beirut 1107 2020, Lebanon. ${ }^{2}$ Weill Cornell Medicine-Qatar- Department of Internal Medicine, Doha, Qatar. ${ }^{3}$ Department of Rheumatology, Medical Institute, Al Ain Hospital, Al Ain, United Arab Emirates. ${ }^{4}$ Dr. Humeira Badsha Medical Center, Rheumatologist City Hospital, Rheumatologist Neurospinal Hospital, Dubai, United Arab Emirates. ${ }^{5}$ Ministry of Health, Muscat Governorate, Oman. ${ }^{6}$ Department of Rheumatology, Cairo University, Giza, Egypt. ${ }^{7}$ Rheumatology Division, Department of Internal Medicine, King Faisal Specialist Hospital and Research Center, Jeddah, Saudi Arabia. ${ }^{8}$ Hamad Medical Corporation, Doha, Qatar. ${ }^{9}$ Aspetar, Doha, Qatar.

${ }^{10}$ Jordan Hospital, Amman, Jordan. ${ }^{11}$ Division of Rheumatology, Department of Medicine, King Saud University, Riyadh, Saudi Arabia. ${ }^{12}$ American University of Beirut, Beirut, Lebanon. ${ }^{13}$ Faculty of Medicine, Univeristé Saint Joseph, Beirut, Lebanon. ${ }^{14}$ Medicine Service and Center for Surgical Medical Acute care Research and Transitions, VA Medical Center, 510, 20th Street South, FOT 805B, Birmingham, AL, USA. ${ }^{15}$ Department of Medicine at School of Medicine, and Division of Epidemiology at School of Public Health, University of Alabama, 1720 Second Ave. South, Birmingham, AL 35294-0022, USA. ${ }^{16}$ Musculoskeletal Statistics Unit, The Parker Institute, Bispebjerg and Frederiksberg Hospital, Copenhagen, Denmark. ${ }^{17}$ Department of Medicine, University of Ottawa, Ottawa, Canada. ${ }^{18}$ Department of Medicine, McMaster University, Hamilton, ON, Canada. ${ }^{19}$ Department of Health Research Methods, Evidence, and Impact (HE\&l), McMaster University, Hamilton, ON, Canada.

${ }^{20}$ Department of Internal Medicine, American University of Beirut, PO Box 11-0236, Riad El Solh, Beirut 1107 2020, Lebanon.

Received: 7 March 2017 Accepted: 7 September 2017

Published online: 21 September 2017

References

1. Steinberg E, Greenfield S, Mancher M, Wolman DM, Graham R. Clinical practice guidelines we can trust. Washington, DC: National Academies Press; 2011.

2. World Health Organization (WHO). WHO handbook for guideline development (Available at http://www.who.int/publications/guidelines/ handbook_2nd_ed.pdf Accessed 12 May 2017). 2014.

3. Fervers B, Burgers JS, Voellinger R, et al. Guideline adaptation: an approach to enhance efficiency in guideline development and improve utilisation. BMJ Qual Saf. 2011;20(3):228-36. doi:10.1136/bmjgs.2010.043257.

4. Harrison $M B$, Légaré $F$, Graham ID, et al. Adapting clinical practice guidelines to local context and assessing barriers to their use. Can Med Assoc J. 2010;182(2):E78-84

5. Darzi A, Abou-Jaoude EA, Agarwal A, et al. Frameworks for adaptation of health guidelines: a methodological survey. J Clin Epidemiol. 2017. doi:10. 1016/j.jclinepi.2017.01.016.

6. Alonso-Coello P, Schunemann HJ, Moberg J, et al. GRADE Evidence to Decision (EtD) frameworks: a systematic and transparent approach to making well informed healthcare choices. 1: Introduction. BMJ. 2016; 353:ARTN i2016. doi:10.1136/bmj.i2016.

7. Schunemann HJ, Wiercioch W, Brozek J, et al. GRADE evidence to decision frameworks for adoption, adaptation and de novo development of trustworthy recommendations: GRADE-ADOLOPMENT. J Clin Epidemiol. 2016; doi:10.1016/j.jclinepi.2016.09.009.

8. Singh JA, Saag KG, Bridges SL, et al. 2015 American College of Rheumatology guideline for the treatment of rheumatoid arthritis. Arthritis Rheumatol. 2016;68(1):1-26.

9. Schünemann HJ, Wiercioch W, Etxeandia I, et al. Guidelines 2.0: Systematic development of a comprehensive checklist for a successful guideline enterprise. Can Med Assoc J. 2014;186(3):E123-E42

10. AMSTAR. Assessing the methodological quality of systematic reviews (Available at https://amstar.ca/Amstar_Checklist.php, accessed on 10 June 2017). 2007.

11. GRADEpro GDT: GRADEpro guideline development too. (Available at: http:// gdt.quidelinedevelopment.org/app/, accessed on 1 Jan 2017) 2017.

12. Guyatt G, Oxman AD, AkI EA, et al. GRADE guidelines: 1. IntroductionGRADE evidence profiles and summary of findings tables. J Clin Epidemiol. 2011;64(4):383-94. https://doi.org/10.1016/j.jclinepi.2010.04.026.

13. Alonso-Coello P, Oxman AD, Moberg J, et al. GRADE Evidence to Decision (EtD) frameworks: a systematic and transparent approach to making well 
informed healthcare choices. 2: Clinical practice guidelines. BMJ. 2016;353: ARTN i2089. https://doi.org/10.1136/bmj.i2089.

14. Stoffer MA, Schoels MM, Smolen JS, et al. Evidence for treating rheumatoid arthritis to target: results of a systematic literature search update. Ann Rheum Dis. 2016;75:16-22.

15. Gaujoux-Viala C, Nam J, Ramiro S, et al. Efficacy of conventional synthetic disease-modifying antirheumatic drugs, glucocorticoids and tofacitinib: a systematic literature review informing the 2013 update of the EULAR recommendations for management of rheumatoid arthritis. Ann Rheum Dis. 2014;73(3):510-5.

16. Menon N, Kothari S, Gogna A, et al. Comparison of intra-articular glucocorticoid injections with DMARDs versus DMARDs alone in rheumatoid arthritis. J Assoc Physicians India. 2014;62(8):673-6.

17. De Cock D, Vanderschueren G, Meyfroidt S, et al. Two-year clinical and radiologic follow-up of early RA patients treated with initial step up monotherapy or initial step down therapy with glucocorticoids, followed by a tight control approach: lessons from a cohort study in daily practice. Clin Rheumatol. 2014;33(1):125-30.

18. Verschueren P, De Cock D, Corluy L, et al. Methotrexate in combination with other DMARDs is not superior to methotrexate alone for remission induction with moderate-to-high-dose glucocorticoid bridging in early rheumatoid arthritis after 16 weeks of treatment: the CareRA trial. Ann Rheum Dis. 2015;74(1):27-34.

19. Scott DL, Ibrahim F, Farewell V, et al. Tumour necrosis factor inhibitors versus combination intensive therapy with conventional disease modifying anti-rheumatic drugs in established rheumatoid arthritis: TACIT noninferiority randomised controlled trial. BMJ. 2015;350:h1046.

20. Heimans L, Wevers-de Boer K, Visser K, et al. A two-step treatment strategy trial in patients with early arthritis aimed at achieving remission: the IMPROVED study. Ann Rheum Dis. 2014;73:1356-61.

21. Schunemann HJ, Woodhead M, Anzueto A, et al. A vision statement on guideline development for respiratory disease: the example of COPD. Lancet. 2009:373(9665):774-9. doi:10.1016/S0140-6736(08)61347-1.

\section{Submit your next manuscript to BioMed Central and we will help you at every step:}

- We accept pre-submission inquiries

- Our selector tool helps you to find the most relevant journal

- We provide round the clock customer support

- Convenient online submission

- Thorough peer review

- Inclusion in PubMed and all major indexing services

- Maximum visibility for your research

Submit your manuscript at www.biomedcentral.com/submit 